\title{
Ethnicity and Second-Line Antihypertensive Medication Response in the ASCOT Trial
}

\author{
Jason T. Davis • Maple M. Fung
}

Published online: 16 November 2010

(C) The Author(s) 2010. This article is published with open access at Springerlink.com

Gupta AK, Poulter NR, Dobson J, et al.: Ethnic differences in blood pressure response to first and second-line antihypertensive therapies in patients randomized in the ASCOT trial. Am J Hypertens 2010, 23:1023-1030.

\section{Rating \\ -Of importance.}

\section{Introduction}

Hypertension, defined as systolic blood pressure (SBP) greater than $140 \mathrm{~mm} \mathrm{Hg}$ or diastolic blood pressure (DBP) greater than $90 \mathrm{~mm} \mathrm{Hg}$, remains a significant problem that results in increased risk of cardiovascular disease [1]. Despite heightened recognition of this problem, less than $40 \%$ of patients are at their goal blood pressure (BP) [2]. There are multiple pharmacologic treatments for hypertension, and it has been known for some time that a patient's likelihood to respond to a particular treatment may depend on that patient's ethnic background [3]. This information has been used to guide initial monotherapy in different racial groups in order to provide optimal treatment, but there is little information regarding combination therapies across different ethnicities. Many patients require multiple

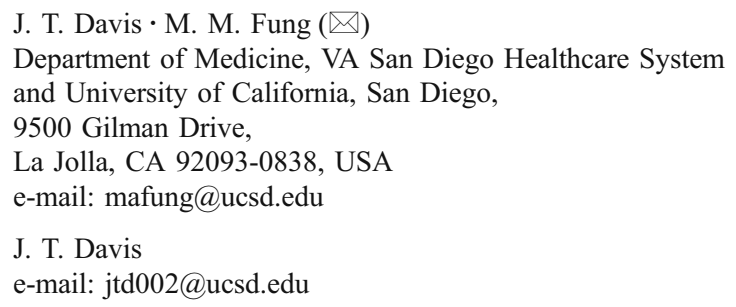

drugs to achieve BP less than 140/90, and some guidelines suggest beginning treatment with a combination of two drugs if the initial BP is more than 20/10 points above goal [4].

The ASCOT (Anglo-Scandinavian Cardiac Outcomes Trial) study evaluated BP reduction in response to monotherapy as well as specific combinations of BP drugs in three different ethnic groups (European origin, African origin, and those from the Indian subcontinent) to evaluate whether a patient's ethnicity plays a role in the outcomes of initial or combination BP therapy.

\section{Aims}

To determine whether BP response in patients during initial monotherapy or during combination treatment with a second-line drug differs by ethnic group (white, black, or South Asian).

\section{Methods}

The study examines data from centers in the United Kingdom involved in the BP-lowering arm of ASCOT, in which 5,425 patients of European (white), African (black), or Indian subcontinent (South-Asian) ancestry were initially randomized to receive monotherapy with either the betaadrenergic antagonist atenolol or the calcium channel blocker (CCB) amlodipine. Of this group, 742 patients changed monotherapy drugs and were not included in the analysis, leaving 4,683 patients for analysis, and 4,168 patients went on to receive dual therapy per study protocol. Only 2,794 patients were included in dual-therapy analysis because of lack of blood pressure logs and changes in medications. Patients who did not achieve targeted BP 
control had a second drug added: the angiotensinconverting enzyme (ACE) inhibitor perindopril was added to the amlodipine group, or the thiazide diuretic bendroflumethiazide was added to the atenolol group. BP was measured at every study visit. The result of monotherapy or BP treated by second-line therapy was measured using the BP on the day of uptitration of treatment to the next level. The final change in BP was measured by subtracting the BP at the beginning of the study from the BP at the end of therapy. Ethnicity was self-determined by a patient questionnaire. Patients were placed into ethnic groups. The study defined whites as individuals of European origin, blacks as individuals of African origin, and South Asians as individuals from the Indian Subcontinent.

The study examined patient response to monotherapy and second-line therapy in all three ethnic groups. Therapy was adjusted in a step-wise fashion per study protocol. Data from patients who switched from one medication arm to another were examined using an intention-to-treat analysis. The study authors used two linear regression models: BP was the dependent variable; ethnicity and allocated treatments were independent variables. The first model controlled for age, gender, body mass index, and years of education. The second model adjusted for these variables as well as duration of dual therapy, previous hypertension treatment, presence of diabetes, and diastolic BP at the start of therapy.

\section{Results}

Of the original 5,425 participants, 5,021 were white, 250 were black, and 154 were South-Asian. Of these, 2,580 were randomized to atenolol and 2,845 to amlodipine; $4,683(86.3 \%)$ of these participants remained on the original drug and were included in analysis. Additional treatment was required by 4,168 patients and was added as described above. In this group, 2,794 continued on the combination regimen and had adequate blood pressure logs.

During monotherapy, all three groups had similar responses to amlodipine. Blacks did not respond as well to atenolol as whites and South-Asians $(P=0.02)$ : the SBP for blacks increased by $4.5 \mathrm{~mm} \mathrm{Hg}$ and there was no change in DBP, compared with decreases of SBP/DBP of 1.6/3.6 $\mathrm{mm} \mathrm{Hg}$ in whites and 4.5/3.6 mm $\mathrm{Hg}$ in SouthAsians. The difference was statistically significant when confounding variables were corrected for in both linear regression models. DBP did not differ between the three groups on atenolol monotherapy $(P=0.02)$.

In response to adding a second-line drug, there were no significant differences across the three ethnic groups when adding bendroflumethiazide to atenolol, prior to adjusting for confounders. When adjusting for confounders in model 1 , blacks had the greatest benefit and South-Asians the least, but statistical significance was lost upon further correction with model 2 . In patients who had perindopril added to amlodipine, there were differences $(P=0.002)$ in reduction of SBP and DBP based on ethnic group: whites had a reduction of $10.2 / 5.6 \mathrm{~mm} \mathrm{Hg}$, blacks $3.2 /$ $2.5 \mathrm{~mm} \mathrm{Hg}$, and South-Asians 11.2/4.8 mm Hg. The change in SBP was statistically significant across groups $(P=0.004)$, but the change in DBP was not.

\section{Discussion}

This study evaluated differences in response to first-line and second-line hypertension treatment in large numbers of patients stratified by ethnicity. The first difference noted was during monotherapy. Black patients responded less well to atenolol than whites and South-Asians, a finding that is consistent with prior reports [3]. Decreased response of black hypertensives to ACE inhibitors has previously been shown in studies examining initial monotherapy [3], but it had not yet been shown after adding an ACE inhibitor as a second-line drug. These findings strengthen the idea of using different antihypertensive drugs in different ethnic groups to provide the most benefit, an idea that has already been documented with initial monotherapy [3] but not with combinations of medicines.

Two editorials accompanying the paper argue that the difference between ethnic groups in their response to antihypertensive treatment may be due in part to differences between the groups in renin levels [5, 6]. Both note that blacks, as defined in ASCOT, tend to have low-renin hypertension compared with whites, and this could be the reason for the difference in response to treatment.

Though some physicians may already be using drugs that have been more effective in monotherapy as a combination, rather than the classic " $\mathrm{AB} / \mathrm{CD}$ " combination tool of pairing either an ACE inhibitor or a beta blocker with a $\mathrm{CCB}$ or a diuretic $[6,7]$, ASCOT is one of the few studies to show that the sequential addition of medications based on ethnicity may be a useful strategy.

\section{Comment}

This paper continues to build on the established idea that antihypertensive therapy should be adjusted based on patient ethnicity. ASCOT expands this concept beyond initial monotherapy to combination therapies. The results also open the door for further study in tailoring multidrug hypertensive therapy.

The study authors suggest that the use of a CCB plus a diuretic may be a more effective combination in black 
patients than a beta blocker plus an ACE inhibitor. This limited conclusion leads to one weakness of the paper, however, which is that only two combinations (diuretic and $\mathrm{CCB}$ vs beta blocker and ACE inhibitor) were studied. Further study is needed before clinical guidelines can be adopted regarding the addition of second-line antihypertensive therapy based on ethnicity. The need for further study is even more apparent now, as recent studies in hypertension that formally examined guidelines obtained from epidemiologic studies and expert opinion in areas such as chronic kidney disease have had surprising results [8]. Also, ASCOT contrasts with the recent ACCOMPLISH trial, which suggests that an ACE inhibitor and a CCB is a more ideal combination, at least in patients at higher risk for cardiovascular events [9]. The ASCOT and ACCOMPLISH trials are not directly comparable, however, as ACCOMPLISH studied ACE inhibitor/ diuretic and $\mathrm{ACE}$ inhibitor/CCB combinations, whereas ASCOT compared an ACE inhibitor/CCB combination versus a beta blocker/diuretic combination. Finally, ASCOT examined BP as the dependent variable, whereas mortality was the focus of ACCOMPLISH.

ASCOT compared one additional ethnic group - SouthAsian ancestry-with whites and blacks. South-Asian patients tended to have an increased response to adding an ACE inhibitor to a $\mathrm{CCB}$, but a decreased response to adding a thiazide diuretic to a beta blocker, though these differences were not statistically significant. There is a possibility of type II error, as a result of the relatively small number of South-Asian patients (only 154 in the entire study, and only 82 going on to dual therapy), so additional investigation will be needed to confirm these findings.

One limitation of ASCOT is that it did not consider comorbid conditions that might suggest forms of BP treatment desirable for indications in addition to BP response alone, such as beta blockers for use in heart failure or ACE inhibitors for patients with chronic kidney disease or a history of myocardial infarction.

This study certainly highlights the need to personalize therapy in order to gain the greatest response to the fewest medications, but self-identified ethnicity may not be an adequate reflection of biogeographic ancestry. Genetic association studies have demonstrated that substantial allelic variation may exist among individuals even within the same self-identified ethnic group. In the United States, genetic admixture in self-proclaimed African Americans may include up to $25 \%$ of alleles derived from European descent [10]. More appropriate may be the investigation of pharmacogenetic-directed therapy. Pharmacogenetic studies in hypertension have so far focused on response to antihypertensive monotherapy. The financial cost and time delay, plus the lack of consistent findings in the literature, have so far limited the clinical contributions of pharmacogenetic therapy to the treatment of hypertension [11].
Continued research may ultimately reveal useful genetic predictors of antihypertensive drug responses.

In this important article, Gupta et al. highlight that ethnic group differences exist in response to both first-line and second-line antihypertensive therapies, suggesting both drugdrug interactions and drug-genetic interactions, which may be quite complex. As evidence-based personalized medicine becomes the goal in clinical medicine, further study is needed to pursue such findings as well as the origin of intergroup differences, with the ultimate goal of optimal treatment of hypertension with minimal adverse effects.

Disclosure Conflicts of Interest: J. Davis: none; M. Fung: research grant received from Forest Laboratories and pending from Takeda Pharmaceuticals.

Open Access This article is distributed under the terms of the Creative Commons Attribution Noncommercial License which permits any noncommercial use, distribution, and reproduction in any medium, provided the original author(s) and source are credited.

\section{References}

1. Lewington S, Clarke R, Qizilbash N, et al.: Age-specific relevance of usual blood pressure to vascular mortality: a meta-analysis of individual data for one million adults in 61 prospective studies. Lancet 2002, 360(9349):1903-13.

2. Meissner I, Whisnant JP, Sheps SG, et al. Detection and control of high blood pressure in the community: do we need a wake-up call? Hypertension 1999, 34:466-471.

3. Materson BJ; Reda DJ; Cushman WC; et al. Single-drug therapy for hypertension in men. A comparison of six antihypertensive agents with placebo. The Department of Veterans Affairs Cooperative Study Group on Antihypertensive Agents. N Engl J Med 1993, 328(13):914-21.

4. Chobanian AV; Bakris GL; Black HR; et al. The Seventh Report of the Joint National Committee on Prevention, Detection, Evaluation, and Treatment of High Blood Pressure: the JNC 7 report. JAMA 2003, 289(19):2560-72.

5. Furberg CD: Renin-guided treatment of hypertension: time for action. Am J Hypertens 2010, 23(9), 929-30.

6. Brown, MJ. Heterogeneity of blood pressure response to therapy. Am J Hypertens 2010, 23 (9), 926-8.

7. National Collaborating Centre for Chronic Conditions, British Hypertension Society. Hypertension: Management of Hypertension in Adults in Primary Care. NICE Clinical Guideline 34 (Update). London, 2006.

8. Appel LJ; Wright JT; Greene T. et al. Intensive Blood-Pressure Control in Hypertensive Chronic Kidney Disease. N Engl J Med 2010, 363:918-929

9. Jamerson K; Weber MA; Bakris GL et al. Benazepril plus amlodipine or hydrochlorothiazide for hypertension in high-risk patients. N Engl J Med 2008, 359(23):2417-2428.

10. Reiner AP; Carlson CS; Ziv E et al. Genetic ancestry, population sub-structure, and cardiovascular disease-related traits among African-American participants in the CARDIA Study. Hum Genet 2007, 121(5):565-75.

11. Mellen PB; Herrington DM. Pharmacogenomics of blood pressure response to antihypertensive treatment. J Hypertens 2005, 23 (7):1311-25. 